\title{
Publisher Correction: Social polymorphism is favoured by the co-evolution of dispersal with social behaviour
}

Charles Mullon (D), Laurent Keller and Laurent Lehmann

Correction to: Nature Ecology \& Evolution https://doi.org/10.1038/s41559-017-0397-y; published online 4 December 2017.

Owing to a technical error, some text was missing in the originally published version of this Article. In the last paragraph of the Discussion section, the second sentence should have read "Yet, the selection that associates dispersal and social behaviour in our model will influence evolution under most ecological settings because it depends only on kin structure, which, due to limited dispersal and the spatial scale of social interactions, is ubiquitous in nature ${ }^{47}$." This error has now been corrected in all versions of the Article.

Published online: 11 December 2017

https://doi.org/10.1038/s41559-017-0439-5 